\title{
Assessment of Endometrial Carcinoma Markers and Hormone Receptors Profile before and after Bariatric Surgery: A Clinico-Pathological-Immunohistochemical Study
}

\author{
Walid Mohamed Elnagar ${ }^{*}$, Samah Said Elbasateeny ${ }^{2,3}$, Mai Mohamed Abdelwahab ${ }^{3}$, \\ Mahmoud Abdou Yassin,, Taha Abdelwahab Baiomy4, Maha Abdelhamid Fathy Amin5, \\ Dalia Abdallah El-Shafei6
}

\author{
${ }^{1}$ Department of Obstetrics and Gynecology, Faculty of Medicine, Zagazig University, Zagazig, Egypt \\ ${ }^{2}$ Department of Pathology, Faculty of Medicine (Rabigh Branch), King Abdul Aziz University, Jeddah, KSA \\ ${ }^{3}$ Department of Pathology, Faculty of Medicine, Zagazig University, Zagazig, Egypt \\ ${ }^{4}$ Department of General Surgery, Faculty of Medicine, Zagazig University, Zagazig, Egypt \\ ${ }^{5}$ Department of Physiology, Faculty of Medicine, Zagazig University, Zagazig, Egypt \\ ${ }^{6}$ Department of Community, Environmental \& Occupational Medicine, Faculty of Medicine, Zagazig University, Zagazig, Egypt \\ Email: *whitewhale1977@gmail.com
}

How to cite this paper: Elnagar, W.M., Elbasateeny, S.S., Abdelwahab, M.M., Yassin, M.A., Baiomy, T.A., Amin, M.A.F. and El-Shafei, D.A. (2019) Assessment of Endometrial Carcinoma Markers and Hormone Receptors Profile before and after Bariatric Surgery: A Clinico-PathologicalImmunohistochemical Study. Open Journal of Obstetrics and Gynecology, 9, 1019-1031. https://doi.org/10.4236/ojog.2019.97099

Received: June 23, 2019

Accepted: July 19, 2019

Published: July 22, 2019

Copyright $\odot 2019$ by author(s) and Scientific Research Publishing Inc. This work is licensed under the Creative Commons Attribution International License (CC BY 4.0).

http://creativecommons.org/licenses/by/4.0/

\section{(C) (i) Open Access}

\begin{abstract}
Background: Obesity is a major risk factor for endometrial carcinoma, and we aim to assess markers of carcinogenesis including PTEN and Ki-67 and hormone receptors profile including ER, $\mathrm{PR}$ and AR before and after bariatric surgery to find out its effects in reducing endometrial carcinoma risk in morbid obese females. Patients and methods: The study included 80 females with morbid obesity (BMI $>40 \mathrm{Kg} / \mathrm{m}^{2}$ ) who underwent bariatric surgery. All were sampled by Pipelle biopsy at baseline and 12 months after operation and examined histopathologically and immunohistochemically for Ki-67, PTEN, ER, PR and AR. Results: Sixty two out of 80 (62/80) females showed no pathological abnormalities; 4 had polyps; 7 had simple endometrial hyperplasia; 4 had atypical endometrial hyperplasia and 3 had endometrial carcinoma. In total, 34 females underwent gastric bypass operation (42.5\%) and 46 underwent a sleeve gastrectomy operation (57.5\%). There was a statistically significant difference between baseline weight and BMI before and after surgery ( $\mathrm{p}$ $<0.001$ ). Of the 7 women with simple hyperplasia, resolution occurred in 5 within 7 months of surgery. Three of 4 females with atypical hyperplasia (AH) showed resolution after 9 months. Mean Ki-67 score was lower at 12
\end{abstract}


months ( $\mathrm{p}<0.001)$ after surgery. 43/77 (55.8\%) baseline biopsies were glandular PTEN null, including $9 / 15$ of the women with baseline endometrial abnormalities, of whom 5/15 regained glandular PTEN expression as their endometrial abnormalities resolved. There was a significant reduction in ER score after surgery $(\mathrm{p}<0.001)$. PR H-scores were not significantly different post-operatively $(\mathrm{p}=0.193)$. AR $\mathrm{H}$-scores were higher significantly in pre-operative biopsies than post-operative ones $(\mathrm{p}<0.001)$. Conclusion: Females with morbid obesity have a higher risk of harboring endometrial abnormalities even if asymptomatic. However, the endometrial pathology and the high ER and PR expression can be normalized within one year without medical treatment, signifying the role of bariatric surgery-induced weight loss in reducing the risk of endometrial neoplasia development. Also, the marked weight loss occurring after bariatric surgery induces highly significant endometrial change as resolution of atypical hyperplasia, and molecular changes as reduction of Ki-67 and restoration of PTEN that are associated with transition of endometrium from high to low risk.

\section{Keywords}

Bariatric, Surgery, Obesity, Endometrial Cancer, Hormone Receptors

\section{Introduction}

Endometrial carcinoma (EC) ranks as the fourth most common cancer in females in the United States with a rising incidence [1].

Obesity is considered as the strongest risk factor for EC and atypical hyperplasia (AH), the precursor lesion [2]. Every $5 \mathrm{~kg} / \mathrm{m}^{2}$ increase in body mass index (BMI) increases risk of EC by 1.6 folds. As a result, females with class III-IV obesity (BMI $\geq 40 \mathrm{~kg} / \mathrm{m}^{2}$ and $\geq 50 \mathrm{~kg} / \mathrm{m}^{2}$, respectively) will have ten folds higher risk of EC compared to normal weight females [3].

It is estimated that $40 \%$ of EC cases are directly resulting from obesity [4]. Also, females with class III-IV obesity who showed long-term weight loss after bariatric surgery had reduction in risk of EC in both retrospective studies [5] [6] and the prospective Swedish Obesity Subjects study [7].

Bariatric surgery can be indicated for patients with clinically morbid obesity $\left(\mathrm{BMI} \geq 40 \mathrm{mg} / \mathrm{m}^{2}\right.$ ) or these with $\mathrm{BMI} \geq 35 \mathrm{mg} / \mathrm{m}^{2}$ but having other co-morbidities as diabetes mellitus, obstructive sleep apnea, obesity-caused cardiomyopathy or severe joint disease [8].

The program of bariatric surgery should include physical activity, dietary counseling, and lifestyle changes after operation [9].

In females with severe obesity, bariatric surgery succeeds in marked weight loss and shows greater long-term benefits compared to non-surgical weight loss methods and it was found that surgical weight loss reduces obesity-related comorbidites and gets better outcomes in severe obese populations [10].

In addition to improved cardiovascular risk factors, there is also reduction on 
cancer risk after bariatric surgery. Studies found a statistically significant reduction in overall cancer risk in females after bariatric surgery [11].

So, there is link between obesity and EC; however, the mechanism is not well understood but may include changes in proliferation markers (PTEN and Ki-67) or excess oestrogen unopposed by progesterone in noncycling premenopausal and postmenopausal endometrium.

The patterns of estrogen receptor (ER) and progesterone receptor (PR) expression in normal endometrial tissue, malignant and premalignant endometria are well characterized. However, their expression in obese women, implications of androgen receptor (AR) expression, and effects of weight reduction on these patterns remain unknown [12].

In this study we hypothesized that weight reduction following bariatric surgery is accompanied by improvement of endometrial morphology and molecular biomarkers involved in endometrial carcinogenesis including PTEN and Ki-67 and normalization of hormone receptors patterns by assessment of ER, PR and AR.

\section{Methods}

\subsection{Study Design}

This is a clinical trial carried out in departments of Obstetrics \& Gynecology, Surgery and pathology, Faculty of Medicine, Zagazig University; on females who underwent bariatric surgery by laparoscopic gastric bypass or sleeve gastrectomy; in the period from January 2016 to January 2019. Our study included women with a BMI $\geq 40 \mathrm{~kg} / \mathrm{m}^{2}$. All participants gave written, informed consent. Approval was obtained from the Research Ethics Committee.

\subsection{Endometrial Sampling and Clinical Management}

Clinical measurements and endometrial samples were obtained at baseline, and 12 months after bariatric surgery. At baseline, we reported the last menstrual period (LMP), menstrual bleeding status. Postmenopausal status was defined as last menses occurring $>1$ year before baseline; otherwise cases were defined as premenopausal. Height and weight were measured using a stadiometer and electronic scales respectively after removal of bulky clothing, and BMI was calculated $\left(\mathrm{kg} / \mathrm{m}^{2}\right)$. Endometrial sampling was performed using a Pipelle $\odot$ (Carefusion, UK) or MedGyn Endo sampler@ (MedGyn, IL). The device was rotated through 360 for sampling the full length of the uterinecavity to get a large, representative sample of the endometrium. The premenopausal cases were sampled during late proliferative phase as possible, while baseline samples were obtained under general anesthesia during bariatric surgery.

Females who showed endometrial pathology were managed after being discussed by Gynecological Oncology Multidisciplinary Team (MDT). Endometrial carcinoma patients underwent total abdominal hysterectomy with bilateral salpingo-oopherectomy followed by adjuvant therapy. While females with atypical 
hyperplasia $(\mathrm{AH})$ had repeated endometrial sampling after 6 - 7 weeks. If abnormalities were persistent, they were treated with 6 months of intrauterine progestin. All women who had resolved endometrial abnormalities were followed by 3- to 6-monthly endometrial observation.

\subsection{Histopathology and Immunohistochemistry}

Endometrial tissue was obtained by pipellebiopsy, formalin-fixed, paraffin embedded, then sectioned and stained by hematoxylin and eosin. It was then examined and assessed by the histopathologist using the WHO classification system [13] [14].

The immunohistochemical staining was carried out using streptavidin-biotin immunoperoxidase technique (Dako-Cytomation, Glostrup, Denmark). Tissues were sectioned at $3-4 \mu \mathrm{m}$ from blocks on positively charged slides then deparaffinized by xylene, and rehydrated in graded alcohol. Then, sections were boiled in buffered citrate ( $\mathrm{pH}$ 6.0), washed with PBS ( $\mathrm{pH}$ 7.3). 6\% $\mathrm{H}_{2} \mathrm{O}_{2}$ in methanol was used to block endogenous peroxidase activity. The slides were incubated overnight with mouse monoclonal Ki-67antibody (Lab Vision, Fremont, CA, USA, 1:50), anti-ER antibody (Clone D07, DAKO, at 1:50), anti-PR antibody (PR 636, DAKO, at 1:50 dilution), PTEN antibody from DAKO (clone 6H2.1, catalog \# M3627, dilution 1:50), mouse monoclonal antihuman AR primary antibody (AR441, Lot No. 10056018, Dako, Santa Clara, CA). The slidesthen were immersed with a biotin-conjugated secondary antibody (Labvision Corporation, Fermont, USA). DAB was used as a chromogen and Mayer's hematoxylin used as a counter stain. After that, the slides were washed with PBS and distilled water.

\subsection{Assessment of Immunohistochemistry}

The Ki-67 score was considered by percentage of glandular cells having positive nuclear staining. Overview of the whole specimen was done to choose 3 representative fields. The final Ki-67 score was determined by $>1000$ nuclei noticed in 3 high-powered fields $(\times 20)$ [15].

ER, PR and AR were assessed using semi-quantitative "H-score". The H-score was the product of the percentage of staining and staining intensity $(0=$ absent, 1 = weak, 2 = moderate, and 3 = intense). The score ranged from $0(100 \%$ unstained) to 300 (100\% intense staining) [16].

PTEN was assessed as "PTEN null" when endometrial glands do not express the protein in the presence of stromal positivity. It was considered as "PTEN positive" if all examined endometrial glands expressed the protein [17].

\subsection{Statistical Analysis}

Quantitative data were expressed as the mean \pm SD \& median (range), and qualitative data were expressed as absolute frequencies (number) \& relative frequencies (percentage). Continuous variables were checked for normality by using 
Shapiro Walk test. Wilcoxon signed ranks test was used to compare two dependent groups of non-normally distributed variables. Paired binary categorical variables were compared using McNemar's test. Paired more than binary categorical variables were compared using McNemar-Bowker's test. All tests were two sided. p-value $<0.05$ was considered statistically significant. All data were collected, tabulated and statistically analyzed using SPSS 20.0 for windows (SPSS Inc., Chicago, IL, USA).

\section{Results}

\subsection{Clinical Baseline Characteristics}

Our study included 80 women with a mean age of 45.08 (range 27 - 66) years; 61 (76.3\%) were pre menopausal. The mean weight was $134 \pm 11.46$, mean BMI was $49.33 \pm 5.38$

Overall, 44 (55\%) had diabetes mellitus, of whom $16(20 \%)$ were on insulin therapy. Regarding symptoms, 55 (68.8\%) had no symptoms, 13 (16.3\%) had abnormal bleeding and, 12 (15\%) had post menopausal bleeding.

Thirty four (34) females underwent gastric bypass operation (42.5\%) and 46 underwent a sleeve gastrectomy operation (57.5\%) (Table 1).

\subsection{Baseline Pathological Findings of Studied Cases}

After histopathological assessment; 62 females showed no pathological abnormalities, 4 had polyps, 7 had simple endometrial hyperplasia, 4 had atypical endometrial hyperplasia and 3 had endometrial carcinoma.

Immunohistochemical (IHC) examinations showed that $56.2 \%$ were PTEN negative, mean Ki-67 was 23.18, mean ER score was $138.22 \pm 84.37$, mean PR score was $146.11 \pm 82.07$ and mean AR score was $73.21 \pm 28.25$ (Table 2).

\subsection{Bariatric Surgery and Changes in Weight}

In total, 34 females underwent gastric bypass (42.5\%) operation and 46 underwent a sleeve gastrectomy operation (57.5\%). The mean weight after 12 months was $93.08 \pm 14.22$, mean BMI was $28.16 \pm 4.64$. There was a statistically significant difference between baseline weight and BMI before and after surgery ( $\mathrm{p}<$ 0.001) (Table 3).

\subsection{Endometrial Pathology, Immunohistochemical Changes before and after Bariatric Surgery}

Of the seven women with simple hyperplasia, resolution occurred in five women within 7 months of bariatric surgery after weight loss of between $12 \%$ and $20 \%$ of their total body weight. In three of them, resolution was accompanied by restoration of normal menstrual cycles. Three of 4 females with AH showed resolution after 9 months (Table 4).

The three females who showed pre-operative endometrial carcinoma, underwent total abdominal hysterectomy. So, on assessment of the Ki-67, PTEN, ER, 
Table 1. Baseline characteristics

\begin{tabular}{|c|c|c|}
\hline \multirow{2}{*}{ Parameters } & \multicolumn{2}{|c|}{$\begin{array}{l}\text { The studied patients } \\
\qquad(\mathrm{N}=80)\end{array}$} \\
\hline & No. & $\%$ \\
\hline \multicolumn{3}{|l|}{$\underline{\text { Age (years) }}$} \\
\hline Mean \pm SD & \multicolumn{2}{|c|}{$45.08 \pm 10.16$} \\
\hline Median (Range) & \multicolumn{2}{|c|}{$46.50(27-66)$} \\
\hline \multicolumn{3}{|l|}{ Weight (kg) } \\
\hline Mean \pm SD & \multicolumn{2}{|c|}{$134 \pm 11.46$} \\
\hline Median (Range) & \multicolumn{2}{|c|}{$132(115-157)$} \\
\hline \multicolumn{3}{|l|}{$\underline{\operatorname{BMI}\left(\mathrm{kg} / \mathrm{m}^{2}\right)}$} \\
\hline Mean \pm SD & \multicolumn{2}{|c|}{$49.33 \pm 5.38$} \\
\hline Median (Range) & \multicolumn{2}{|c|}{$49(41-59)$} \\
\hline \multicolumn{3}{|l|}{$\underline{\text { Parity }}$} \\
\hline Nulliparous & 14 & $17.5 \%$ \\
\hline One & 21 & $26.3 \%$ \\
\hline Two & 28 & $35 \%$ \\
\hline Three & 17 & $21.3 \%$ \\
\hline \multicolumn{3}{|l|}{ Menopausal status } \\
\hline Premenopausal & 61 & $76.3 \%$ \\
\hline Postmenopausal & 19 & $23.8 \%$ \\
\hline \multicolumn{3}{|l|}{$\underline{\text { Symptoms }}$} \\
\hline Asymptomatic & 55 & $68.8 \%$ \\
\hline Abnormal uterine bleeding & 13 & $16.3 \%$ \\
\hline Postmenopausal bleeding & 12 & $15 \%$ \\
\hline \multicolumn{3}{|l|}{$\underline{\text { Diabetes mellitus }}$} \\
\hline No & 36 & $45 \%$ \\
\hline Yes & 44 & $55 \%$ \\
\hline \multicolumn{3}{|l|}{$\underline{\text { DM medications }}$} \\
\hline Insulin & 16 & $20 \%$ \\
\hline Metformin & 22 & $27.5 \%$ \\
\hline Other oral hypoglycemic & 7 & $8.8 \%$ \\
\hline \multicolumn{3}{|l|}{ Bariatric surgery } \\
\hline Bypass & 34 & $42.5 \%$ \\
\hline Sleeve & 46 & $57.5 \%$ \\
\hline
\end{tabular}

$\mathrm{PR}$, and AR scores, the number became 77. Compared to baseline, mean Ki-67 score was lower at 12 months $(\mathrm{p}<0.001) 22.59 \pm 5.6714 .06 \pm 6.17$ after bariatric surgery. 
Table 2. Baseline pathological findings.

\begin{tabular}{|c|c|c|}
\hline \multirow{2}{*}{ Parameters } & \multicolumn{2}{|c|}{$\begin{array}{l}\text { The studied patients } \\
\qquad(\mathrm{N}=80)\end{array}$} \\
\hline & No. & $\%$ \\
\hline \multicolumn{3}{|l|}{ Morphology } \\
\hline Normal & 62 & $77.5 \%$ \\
\hline Polyp & 4 & $5 \%$ \\
\hline Simple endometrial hyperplasia & 7 & $8.8 \%$ \\
\hline Atypical endometrial hyperplasia & 4 & $5 \%$ \\
\hline EEC grade I & 3 & $3.8 \%$ \\
\hline \multicolumn{3}{|l|}{$\underline{\text { PTEN }}$} \\
\hline Negative & 45 & $56.2 \%$ \\
\hline Positive & 35 & $43.8 \%$ \\
\hline \multicolumn{3}{|l|}{$\underline{\mathrm{Ki}-76(\%)}$} \\
\hline Mean \pm SD & \multicolumn{2}{|c|}{$23.18 \pm 6.34$} \\
\hline Median (Range) & \multicolumn{2}{|c|}{$22(11-42)$} \\
\hline \multicolumn{3}{|l|}{$\underline{\text { ER H-score }}$} \\
\hline Mean \pm SD & \multicolumn{2}{|c|}{$138.22 \pm 84.37$} \\
\hline Median (Range) & \multicolumn{2}{|c|}{$129(24-290)$} \\
\hline \multicolumn{3}{|l|}{$\underline{\text { PR H-score }}$} \\
\hline Mean \pm SD & \multicolumn{2}{|c|}{$146.11 \pm 82.07$} \\
\hline Median (Range) & \multicolumn{2}{|c|}{$140(9-310)$} \\
\hline \multicolumn{3}{|l|}{$\underline{\text { AR H-score }}$} \\
\hline Mean \pm SD & \multicolumn{2}{|c|}{$73.21 \pm 28.25$} \\
\hline Median (Range) & \multicolumn{2}{|c|}{$70(26-125)$} \\
\hline
\end{tabular}

Table 3. Comparison between baseline and 12 month postoperative weight and BMI.

\begin{tabular}{cccc}
\hline Parameters & $\begin{array}{c}\text { Baseline } \\
(\mathrm{N}=80)\end{array}$ & $\begin{array}{c}12 \text { month postoperative } \\
(\mathrm{N}=80)\end{array}$ & p-value \\
\hline Weight $(\mathrm{kg})$ & $134 \pm 11.46$ & $93.08 \pm 14.22$ & \\
Mean \pm SD & $132(115-157)$ & $90(65-130)$ & $<0.001$ \\
Median (Range) & & & \\
BMI $\left(\mathrm{kg} / \mathrm{m}^{2}\right)$ & $49.33 \pm 5.38$ & $28.16 \pm 4.64$ & $<0.001$ \\
Mean \pm SD & $49(41-59)$ & $28(20-40)$ & \\
Median (Range) & & & \\
\hline
\end{tabular}

Continuous variables were expressed as mean \pm SD \& median (range); $\bullet$ Wilcoxon signed ranks test.

The median $\mathrm{H}$-score of ER for patients with pre-operative hyperplasia was 220, which was significantly higher than for patients without pre-operative hyperplasia $(165, \mathrm{p}=0.001)$. Patients without pre-operative pathology had no 
Table 4. Comparison between baseline and 12 monthspost-operative morphology.

\begin{tabular}{ccccccc}
\hline & & \multicolumn{4}{c}{ Morphology 12 months postoperative } & \\
\cline { 3 - 5 } $\begin{array}{c}\text { Morphology } \\
\text { Baseline }\end{array}$ & & Normal & Polyp & $\begin{array}{c}\text { Simple } \\
\text { endometrial } \\
\text { hyperplasia }\end{array}$ & $\begin{array}{c}\text { Atypical } \\
\text { endometrial } \\
\text { hyperplasia }\end{array}$ & Total \\
Normal & No. & 60 & 0 & 2 & 0 & 62 \\
& $\%$ & $77.9 \%$ & $0 \%$ & $2.6 \%$ & $0 \%$ & $80.5 \%$ \\
Polyp & No. & 4 & 0 & 0 & 0 & 4 \\
Simple endometrial & No. & 5 & 2 & 0 & $0 \%$ & $5.2 \%$ \\
hyperplasia & $\%$ & $6.5 \%$ & $2.6 \%$ & $0 \%$ & $0 \%$ & 7 \\
Atypical endometrial \\
hyperplasia
\end{tabular}

significant change in their ER H-scores after bariatric surgery (144 to 172 respectively, $\mathrm{p}=0.22$ ), while the ER H-scores of patients with hyperplasia dropped more significantly ( 251 to $128, \mathrm{p}=0.03$ ).

Totally, there was a significant reduction in ER score after surgery $(\mathrm{p}<0.001)$.

PR H-scores were higher also for patients with pre-operative hyperplasia (median 254) when compared to those without hyperplasia (180, $\mathrm{p}=0.001)$. Patients with pre-operative hyperplasia showed a mean drop in $\mathrm{H}$-score of 108 , while those without hyperplasia had a mean rise of $3(\mathrm{p}=0.06)$. Follow-up PR H-scores were not significantly different post-operatively $(146.54 \pm 82.05$ vs $123.89 \pm$ $71.89, \mathrm{p}=0.193)$.

AR $\mathrm{H}$-scores were higher significantly in pre-operative biopsies than post-operative ones (median 70 vs $50, \mathrm{p}<0.001$ ) (Table 5).

In total, 43/77 (55.8\%) baseline biopsies were glandular PTEN null, including 9/15 of the women with baseline endometrial abnormalities, of whom 5/15 regained glandular PTEN expression as their endometrial abnormalities resolved. 34 females were PTEN positive at baseline, but after surgery, 26 only became PTEN null $(\mathrm{p}=0.002)($ Table 6$)$.

\section{Discussion}

Bariatric surgery is associated with a considerable and a clinical significant reduction of uterine malignancy risk. Our study, in concurrence with the results of others, has revealed that the uterine malignancy risk increases with increasing BMI [18] [19] [20] [21]. So, our finding with previous ones, suggest that obesity can be considered as a modifiable risk factor for endometrial carcinoma.

The pathogenic mechanisms of obesity associated endometrial carcinoma include exogenous estrogen formation and insulin resistance. It is well recognized 
Table 5. Comparison between baseline and 12 months postoperative pathological findings.

\begin{tabular}{|c|c|c|c|c|c|}
\hline \multirow{2}{*}{ Parameters } & \multicolumn{2}{|c|}{$\begin{array}{l}\text { Baseline } \\
(\mathrm{N}=77)\end{array}$} & \multicolumn{2}{|c|}{$\begin{array}{l}12 \text { month postoperative } \\
\qquad(\mathrm{N}=77)\end{array}$} & \multirow{2}{*}{$\mathrm{p}$-value } \\
\hline & No. & $\%$ & No. & $\%$ & \\
\hline \multicolumn{6}{|l|}{ Morphology } \\
\hline Normal & 62 & $80.5 \%$ & 72 & $93.5 \%$ & \multirow{3}{*}{$0.036 \S$} \\
\hline Polyp & 4 & $5.2 \%$ & 2 & $2.6 \%$ & \\
\hline Simple endometrial hyperplasia & 7 & $9.1 \%$ & 2 & $2.6 \%$ & \\
\hline Atypical endometrial hyperplasia & 4 & $5.2 \%$ & 1 & $1.3 \%$ & \\
\hline \multicolumn{6}{|l|}{$\underline{\text { PTEN }}$} \\
\hline Negative & 43 & $55.8 \%$ & 27 & $31.2 \%$ & \multirow{2}{*}{$0.002 \dagger$} \\
\hline Positive & 34 & $44.2 \%$ & 26 & $68.8 \%$ & \\
\hline \multicolumn{6}{|l|}{$\underline{\mathrm{Ki}-76(\%)}$} \\
\hline Mean \pm SD & 22. & 5.67 & & $=6.17$ & \multirow{2}{*}{$<0.001 \bullet$} \\
\hline Median (Range) & & 34) & & $-40)$ & \\
\hline \multicolumn{6}{|l|}{$\underline{\text { ER H-score }}$} \\
\hline Mean \pm SD & 140. & 84.55 & 115 & $=63.14$ & \multirow{2}{*}{$<0.001 \bullet$} \\
\hline Median (Range) & 137 & 290) & 110 & - 260) & \\
\hline \multicolumn{6}{|l|}{$\underline{\text { PR H-score }}$} \\
\hline Mean \pm SD & 146. & 82.05 & & 71.89 & \multirow{2}{*}{$0.193 \bullet$} \\
\hline Median (Range) & 140 & 310) & 106 & - 359) & \\
\hline \multicolumn{6}{|l|}{$\underline{\text { AR H-score }}$} \\
\hline Mean \pm SD & 72.4 & 8.22 & & 26.20 & \multirow{2}{*}{$<0.001 \bullet$} \\
\hline Median (Range) & 70 & 125) & & 107) & \\
\hline
\end{tabular}

Categorical variables were expressed as number (percentage); Continuous variables were expressed as mean \pm SD \& median (range); $₫$ McNemar-Bowker’s test; $\uparrow$ McNemar’s test; $\bullet$ Wilcoxon signed ranks test.

Table 6. Comparison between baseline and 12 month postoperative PTEN.

\begin{tabular}{lcccc}
\hline \multirow{2}{*}{$\begin{array}{c}\text { PTEN } \\
\text { Baseline }\end{array}$} & & \multicolumn{2}{c}{ PTEN 12 months postoperative } & \multirow{2}{*}{ Total } \\
\cline { 3 - 4 } Negative & No. & Negative & Positive & \\
\hline \multirow{2}{*}{ Positive } & $\%$ & 16 & 27 & 43 \\
& No. & $20.8 \%$ & $35.1 \%$ & $55.8 \%$ \\
& $\%$ & 8 & 26 & 34 \\
Total & No. & $10.4 \%$ & $33.8 \%$ & $44.2 \%$ \\
& $\%$ & 24 & 53 & 77 \\
& & $31.2 \%$ & $68.8 \%$ & $100 \%$ \\
\hline
\end{tabular}

that circulating estradiol levels are affected by the percentage of adipose tissue, as aromataseenzyme is found in the adipose tissue. Also, obesity induce a chronic 
inflammation state, with increasing levels of interleukin-6, C-reactive protein and TNF- $\alpha$, leading to insulin resistance, which is linked to low levels of sex hormone binding globulin, with subsequent increasing in the bioavailability of estrogen, and is related to tumorogenesis and tumor spread [22].

The connection of cancer risk reduction with bariatric surgery has been already established [23] [24]. Bariatric surgery cause weight loss throughout decreased caloric intake and increased physical activity. It also decreases cancer risk by many weight loss independent pathways [25]. These mechanism include reorganization of gastrointestinal anatomy, decreased iinflammatory markers and inflammation, changes in bile and fat metabolism, gut hormone release, and alternation of intestinal gluconeogenesis [23].

We have found a high frequency of endometrial pathology in class III-IV obese females selected for gastric bypass or sleeve gastrectomy. Also, occult $\mathrm{AH}$ and EC were seen in a part of our participants at baseline, Bariatric surgery resulted in significant reduction in weight and fall in BMI and resolution of some $\mathrm{AH}$ cases. This finding support the causal association between obesity and endometrial carcinoma.

Our study revealed that class III-IV obesity is associated with high Ki-67, and loss of PTEN expression. This pre-proliferative profile, act as background for mutational changes that initiate endometrial carcinogenesis [26].

In addition, this combined expression profile of these biomarkers give a emolecular signature denoting the endometrium at risk, and supply a chance for screening, risky women for the prevention of EC.

In females with endometrial pathology, loss of glandular PTEN was common finding at baseline biopsy and its restoration accompanied resolution of atypical hyperplasia. In women with normal endometrial biopsy, baseline PTEN null persisted through weight loss, this suggests that bariatric surgery is effective in clearing these endometrial precursor lesions. Consequently, PTEN loss can be a reversible element of early endometrial carcinogenesis and its regain indicates the conversion to low risk endometrium [27].

A drop of Ki-67 was noticed at 12 months after bariatric surgery. This was noticed in most cases with endometrial abnormalities who performed repeat sampling, Ki-67 is a well known prognostic marker for EC, and it is suggested that a drop of Ki-67 is linked to EC risk reduction [28].

Another important finding in our study is that ER, PR and AR scores of females with hyperplasia dropped after bariatric surgery without any medications. This may indicate a direct consequence of weight loss on peripheral estrogen secretion, loss of gastrointestinal adipokines due to excision of part of intestine or the restoration of ovulation with subsequent effect of endogenous progesterone. However, morbidly obese females are relatively hyper estrogenic, so, the mechanism by which weight reduction act may simply be adjustment of the estrogen and progesterone balance via estrogen mitigation. Estrogen is recognized to persuade proliferation and increased of ER and PR expression, which are usually over-expressed in type I, obesity-related endometrial carcinoma; while PR un- 
dergo down-regulation [29].

We cannot exclude sampling errors as a source of errors. We decided the Pipelle biopsy method over curettage for our study, to minimize surgical risks inan volunteer asymptomatic females; nevertheless, this may not be the optimal method for evaluation, and despite that most studies found a high degree of correlation between its results and curettage results, there may be increasing discrepancies among abnormal specimens, which may be significant in a study as ours.

We also recommend further studies with larger sample size and use of control matched groups for better results evaluation.

\section{Conclusion}

Females with morbid obesity have a higher risk of harboring endometrial abnormalities even if asymptomatic. However, the endometrial pathology and the high ER and PR expression can be normalized within one year without medical treatment, signifying the role of bariatric surgery-induced weight loss in reducing the risk of endometrial neoplasia development. Also, the marked weight loss occurring after bariatric surgery induces highly significant endometrial change as resolution of atypical hyperplasia, and molecular changes as reduction of Ki-67 and restoration of PTEN that are associated with transition of endometrium from high to low risk.

\section{Conflicts of Interest}

The authors declare no conflicts of interest regarding the publication of this paper.

\section{References}

[1] Uterine Cancer Statistics (2015) Cancer Research UK. http://www.cancerresearchuk.org/health-professional/cancer-statistics/statistics-bycancer-type/uterine-cancer

[2] Linkov, F., Edwards, R., Balk, J., Yurkovetsky, Z., Stadterman, B., Lokshin, A. and Taioli, E. (2008) Endometrialhyperplasia, Endometrial Cancer and Prevention:gaps in Existing Research of Modifiable Risk Factors. European Journal of Cancer, 44, 1632-1644. https://doi.org/10.1016/j.ejca.2008.05.001

[3] Crosbie, E., Zwahlen, M., Kitchener, H., Egger, M. and Renehan, A.G. (2010) Body Mass Index, Hormone Replacement Therapy and Endometrial Cancer Risk: A Meta Analysis. Cancer Epidemiology, Biomarkers \& Prevention, 19, 3119-3130. https://doi.org/10.1158/1055-9965.EPI-10-0832

[4] Arnold, M., Pandeya, N., Byrnes, G., et al. (2015) Global Burden of Cancer Attributable to High Body-Mass Index in 2012: A Population-Based Study. The Lancet Oncology, 16, 36-46. https://doi.org/10.1016/S1470-2045(14)71123-4

[5] Adams, T.D., Stroup, A.M., Gress, R.E., et al. (2009) Cancer Incidence and Mortality after Gastric Bypass Surgery. Obesity, 17, 796-802. https://doi.org/10.1038/oby.2008.610

[6] Ward, K.K., Roncancio, A.M., Shah, N.R., et al. (2014) Bariatric Surgery Decreases the Risk of Uterine Malignancy. Obstetrics \& Gynecology, 133, 63-66. 
[7] Sjöström, L., Gummesson, A., Sjöström, C.D., et al. (2009) Effects of Bariatric Surgery on Cancer Incidence in Obese Patients in Sweden (Swedish Obese Subjects Study): A Prospective, Controlled Intervention Trial. The Lancet Oncology, 10, 653-662. https://doi.org/10.1016/S1470-2045(09)70159-7

[8] (1992) Gastrointestinal Surgery for Severe Obesity: National Institutes of Health Consensus Development Conference Statement. American Journal of Clinical $\mathrm{Nu}$ trition, 9, 615S-619S.

[9] National Institute of Health (2000) The Practical Guide: Identification, Evaluation, and Treatment of Overweight and Obesity in Adults.

[10] Nelson, D., Porta, R., Blair, K., Carter, P. and Martin, M. (2012) The Duodenal Switch for Morbid Obesity: Modification of Cardiovascular Risk Markers Compared with Standard Bariatric Surgeries. The American Journal of Surgery, 203, 603-608. https://doi.org/10.1016/j.amjsurg.2011.12.004

[11] Christou, N.V., Lieberman, M., Sampalis, F. and Sampalis, J.S. (2008) Bariatric Surgery Reduces Cancer Risk in Morbidly Obese Patients. Surgery for Obesity and Related Diseases, 4, 691-695. https://doi.org/10.1016/j.soard.2008.08.025

[12] Trimble, C.L., Method, M., Leitao, M., Lu, K., Ioffe, O., Hampton, M., et al. (2012) Society of Gynecologic Oncology Clinical Practice Committee. Obstetrics \& Gynecology, 120, 1160-1175.

[13] Emons, G., Beckmann, M.W., Schmidt, D., et al. (2015) Uterus Commission of the Gynecologicaloncology Working Group (AGO). New WHO Classification of Endometrial Hyperplasias. Geburtshilfe Frauenheilkd, 75, 135-136. https://doi.org/10.1055/s-0034-1396256

[14] Kurman, R.J., Carcangiu, M., Herrington, C.S., et al. (2014) WHO Classification of Tumours of Female Reproductive Organs. 4th Edition, IARC, Lyon.

[15] Dowsett, M., Nielsen, T.O., A’Hern, R., et al. (2011) Assessment of Ki67 in Breast Cancer: Recommendations from the International Ki67 in Breast Cancer Working Group. JNCI: Journal of the National Cancer Institute, 103, 1656-1664.

[16] Argenta, P., Svendsen, C., Elishaev, E., Gloyeske, N., et al. (2014) Hormone Receptor Expression Patterns in the Endometrium of Asymptomatic Morbidly Obese Women before and after Bariatric Surgery. Gynecologic \& Oncology, 133, 78-82.

[17] Orbo, A., Rise, C.E. and Mutter, G.L. (2006) Regression of Latent Endometrial Precancers by Progestin Infiltrated Intrauterine Device. Cancer Research, 66, 5613-5617. https://doi.org/10.1158/0008-5472.CAN-05-4321

[18] Ward, K.K., Roncancio, A.M., Shah, N.R., Davis, M.A., Saenz, C.C., McHale, M.T. and Plaxe, S.C. (2013) The Risk of Uterine Malignancy Is Linearly Associated with Body Mass Index in a Cohort of US Women. American Journal of Obstetrics \& Gynecology, 209, 579.e1-579.e5.

[19] Wolin, K.Y., Carson, K. and Colditz, G.A. (2010) Obesity and Cancer. The Oncologist, 15, 556-565. https://doi.org/10.1634/theoncologist.2009-0285

[20] Yang, T.Y., Cairns, B.J., Allen, N., Sweetland, S., Reeves, G.K. and Beral, V. (2012) Postmenopausal Endometrial Cancer Risk and Body Size in Early Life and Middle Age: Prospective Cohort Study. British Journal of Cancer, 107, 169-175. https://doi.org/10.1038/bjc.2012.229

[21] Jeong, N.H., Lee, J.M., Lee, J.K., Kim, J.W., Cho, C.H., Kim, S.M., et al. (2010) Role of Body Mass Index as a Risk and Prognostic Factor of Endometrioid Uterine Cancer in Korean Women. Gynecologic \& Oncology, 118, 24-28.

[22] Verma, M. (2009) Cancer Epidemiology. In: Methods of Molecular Biology, 57-88, Springer Nature, Switzerland. 
[23] Ashrafian, H., Ahmed, K., Rowland, S.P., Patel, V.M., Gooderham, N.J., Holmes, E., Darzi, A. and Athanasiou, T. (2011) Metabolic Surgery and Cancer: Protective Effects of Bariatric Procedures. Cancer, 117, 1788-1799.

[24] Ward, K.K., Shah, N.R., Saenz, C.C., McHale, M.T., Alvarez, E.A. and Plaxe, S.C. (2012) Cardiovascular Disease Is the Leading Cause of Death among Endometrial Cancer Patients. Gynecologic \& Oncology, 126, 176-199.

[25] Bilhorn, K.R., Luo, Y., Lee, B.T. and Wong, N.D. (2012) High-Density Lipoprotein Cholesterol, High Sensitivity C-Reactive Protein, and Cardiovascular Disease in United States Adults. The American Journal of Cardiology, 110, 1464-1467. https://doi.org/10.1016/j.amjcard.2012.06.056

[26] Sanderson, P.A., Critchley, H.O.D., Williams, A.R., Arends, M.J. and Saunders, P.T. (2017) New Concepts for an Old Problem: The Diagnosis of Endometrial Hyperplasia. Human Reproduction Update, 23, 232-254.

[27] Ørbo, A., Arnes, M., Lyså, L.M. and Straume, B. (2015) Expression of PAX2 and PTEN Correlates to Therapy Response in Endometrial Hyperplasia. Anticancer Research, 35, 6401-6409.

[28] Kitson, S., Sivalingam, V.N., Bolton, J., et al. (2017) Ki-67 in Endometrial Cancer: Scoring Optimization and Prognostic Relevance for Window Studies. Modern Pathology, 30, 459-468.

[29] Fader, A.N., Nieves Arriba, L., Frasure, H.E. and von Gruenigen, V.E. (2009) Endometrial Cancer and Obesity: Epidemiology, Biomarkers, Prevention and Survivorship. Gynecologic \& Oncology, 114, 121-127.

https://doi.org/10.1016/j.ygyno.2009.03.039 